\title{
Transforming Service Delivery in Government through Integration of Web 3.0 and Social Media Technologies: A Case for Developing Countries
}

\author{
Josphat Karani Mwai ${ }^{1, *}$, Simon Maina Karume ${ }^{2}$, John W Makokha ${ }^{3}$ \\ ${ }^{1}$ Department of Information Technology, Kibabii University, Kenya \\ ${ }^{2}$ Department of Information Technology, Laikipia University, Kenya \\ ${ }^{3}$ Department of Science Technology and Engineering, Kibabii University, Kenya
}

Received May 26, 2020; Revised August 3, 2020; Accepted August 10, 2020

\section{Cite This Paper in the following Citation Styles}

(a): [1] Josphat Karani Mwai, Simon Maina Karume, John W Makokha , "Transforming Service Delivery in Government through Integration of Web 3.0 and Social Media Technologies: A Case for Developing Countries," Computer Science and Information Technology, Vol. 8, No. 3, pp. 57 - 65, 2020. DOI: 10.13189/csit.2020.080301.

(b): Josphat Karani Mwai, Simon Maina Karume, John W Makokh (2020). Transforming Service Delivery in Government through Integration of Web 3.0 and Social Media Technologies: A Case for Developing Countries. Computer Science and Information Technology, 8(3), 57 - 65. DOI: 10.13189/csit.2020.080301.

Copyright $\bigcirc 2020$ by authors, all rights reserved. Authors agree that this article remains permanently open access under the terms of the Creative Commons Attribution License 4.0 International License

\begin{abstract}
This study focuses on how to integrate Social Media and Web 3.0 features within government portals for personalised service delivery within government portals. The study was based on the Kenya government national portal (eCitizen) and sought the views of 94 experts responsible for managing social media and portal service on the eCitizen portal. The study employed Factor Analysis to analyse the experts' responses. Through factor analysis, the study identified six core factors that make up a Personalised Integrated Service Delivery (PISD) framework. These factors were services, web 3.0 features, social media management, security, one-stop-shop for government services and external factors. The study also described a Personalised Integrated System Architecture based on the PISD framework. The PISD framework and proposed system architecture can be used to guide the integration of social media and web 3.0 features within government portals for Personalised Integrated Service Delivery to citizens especially for developing countries like Kenya.
\end{abstract}

Keywords Personalised Service Delivery within Government Portals, Government Portals Services, eServices from Government Portals, Citizen Centred Government Services

\section{Introduction}

According to the United Nations e-Government Survey 2018 (a bi-annual survey) [1] one of the most current trends in e-Government is the advancement of people-driven services (personalised service). The UN points out that governments should address the growing demand for more personalised services that reflect individual needs, as well as people's aspiration to be more closely engaged in the design and delivery of services. These new demands can transform the way the public sector operates [2]. The United Nations (2016) report also emphasises the importance of having integrated services as a key success factor of any e-government initiative. According to the report, another new trend in e-government is the evolution towards integrated public services online through one-stop platforms (web portals). The UN report emphasises the need for personalised integrated service delivery as a key component of any government portal. The need for personalised integrated service delivery as expressed in the UN report forms the basis of this study.

The objectives of this study were: to develop a framework for Personalised Integrated Service Delivery within government portals and to describe the system architecture for a Personalised Integrated Service Delivery environment based on the framework developed in objective one. To achieve this, the study recommends the 
integration of social media and Web 3.0 features. This paper begins by describing the methodology used in this research and then discusses the results of the study. Finally, it describes system architecture for implementing a Personalised Integrated Service Delivery (PISD) framework within government portals.

\section{Methodology}

This study adopted Design Science Research (DSR) design. DSR design was selected because it supports collaboration between industry and academia in the development of new artifacts or innovate artifacts for solving problems or improving facilities in order to create a new reality. The study was based on the Kenyan government official portal (eCitizen). The target population for the study comprised of (94) experts managing the eCitizen portal. These experts were drawn from the teams managing social media accounts and portal services in all the 10 organizations which are part of the eCitizen portal, Kenya's official national portal [3]. Census sampling was used because the target population of 94 experts was not very large and it was possible to gather data from every member of the target population.

Research instruments used in this study were questionnaires and guided interviews. These instruments were used to collect data regarding how social media and web 3.0 technologies were being used to enhance service delivery by the government of Kenya. The instruments were also used to collect data on factors which could improve the integration of social media and web 3.0 tools within government portals. This is geared towards Personalised Integrated Service Delivery to citizens. Quality control of these instruments was done to establish their validity and reliability. The reliability of the instruments was established through pretesting and piloting. Cronbach's alpha of 0.791 value was calculated for 19 items. Thereafter, the tool was deemed reliable for use in the study.

\section{Response Rate}

The target population for this research comprised of the teams managing social media accounts and portal services in all the 10 organizations which are part of eCitizen portal, Kenya's official national portal. The response rate of the study was 72 out 94 respondents which is a $76.6 \%$ response rate. This response rate was found to be sufficient and representative. The study used a questionnaire and guided interview to collect the views of the experts responsible for developing and managing the services offered through the Kenya government eCitizen portal and social media accounts on this portal.

The study sought to investigate the following issues:

i) What is the role of Web 3.0 and Social Media technologies in government portals?

ii) What factors are necessary for the integration of Web 3.0 and Social Media technologies in government portals?

iii) How can a framework for integrating Web 3.0 and Social Media technologies in government portals for personalised integrated services delivery be developed?

\section{Results and Discussion}

Data was collected and analysed using SPSS. Descriptive analysis highlighted twenty-seven (27) variables that the experts indicated are important components for personalised integrated service delivery to be achieved within government portals. The study used factor analysis to condense the many variables identified through descriptive analysis into a few key factors that would constitute the Personalized Integrated Service Delivery (PISD) framework. Exploratory Factor Analysis (EFA) technique was used to identify the key factors necessary for personalised service delivery within government portals based on the results obtained from the descriptive initial analysis of the data collected. Through factor analysis, twenty-seven (27) variables were extracted as illustrated in table 1 below. These twenty-seven variables are the ones that were highlighted by the experts as essential components for the integration of social media and web 3.0 technologies within government portals.

The next step of the study was consolidating the extracted variables into a few key factors that would constitute the Personalized Integrated Service Delivery (PISD) framework. The cumulative percentage of variance was extracted and Kaiser's criteria (eigenvalue > 1 rule) [4] was employed for factor reduction and retention. This reduced the 27 variables to seven (7) factors. The 7 factors retained were the ones with eigenvalue $>1$ as recommended by the Kaiser rule on factor retention. After this, the study used the Varimax method which is one of the orthogonal methods for factor rotation. The goal of Factor rotation was to clearly establish which variables load on to each of the seven factors retained, as illustrated in table 1 . 
Table 1. Extracted variables and Rotated Component Matrix table

\begin{tabular}{|c|c|c|c|c|c|c|c|}
\hline \multirow{2}{*}{ Name of Variable } & \multicolumn{7}{|c|}{ Factor } \\
\hline & 1 & 2 & 3 & 4 & 5 & 6 & 7 \\
\hline campaign /promotion & .883 & & & & & & \\
\hline marketing & .871 & & & & & & \\
\hline address customer complaints & .822 & & & & & & \\
\hline Survey & .749 & & & & & & \\
\hline information dissemination & .740 & & & & & & \\
\hline predict a service & & .888 & & & & & \\
\hline intelligent services & & .852 & & & & & \\
\hline integrated services & & .761 & & & & & \\
\hline select delivery platform & & .726 & & & & & \\
\hline recognize users & & .588 & & & & & \\
\hline automated services & & .555 & & & & & \\
\hline personalization & & .527 & & & & & \\
\hline respond or act upon issues & & & .915 & & & & \\
\hline store social media profile data & & & .895 & & & & \\
\hline reject/remove inappropriate content & & & .845 & & & & \\
\hline collect and store sm data & & & .806 & & & & \\
\hline dedicated team & & & .504 & & & & \\
\hline public participation & & & & .718 & & & \\
\hline transactional services & & & & .525 & & & \\
\hline secure services & & & & & .525 & & \\
\hline integration of government data & & & & & & .621 & \\
\hline device independent services & & & & & & .666 & \\
\hline one-stop-shop for services & & & & & & .588 & \\
\hline social media policy & & & & & & & .570 \\
\hline training & & & & & & & .528 \\
\hline institutional culture & & & & & & & .694 \\
\hline \multicolumn{8}{|c|}{$\begin{array}{l}\text { Extraction Method: } \\
\text { Principal Component Analysis } \\
\text { Rotation Method: Varimax with Kaiser Normalization }\end{array}$} \\
\hline a. Rotation converged in 11 iteration & & & & & & & \\
\hline
\end{tabular}

After factor extraction, factor retention and factor rotation, the last step in factor analysis is interpretation. This process involves the examination of select variables which are attributable to a construct (factor) and allocating a name for that factor. The labelling of a construct is a theoretical, subjective and inductive process [5]. The researcher was careful to select labels that were significant and reflected the theoretical and conceptual intent.

From the factor rotation table 1 above all the variables loading onto factor one (1) and factor four (4) have a common theme around the type of services that can be offered through social media. Therefore, all the variables under these two factors ( 1 and 2) were collectively named services and form the first sub-construct of the Personalized Integrated Service Delivery (PISD) framework. The sub-construct (service) was made up of these variables; campaign/promotion services with a factor loading of (.883), marketing (.871), address customer complaints (.822), survey (.749), and information dissemination services (.740) all load together on factor one (1) and public participation with a loading of (.718) and transactional services (.525) both loading on factor four (4).

The second sub-construct identified as part of Personalized Integrated Service Delivery (PISD) framework was Web 3.0 features. The sub-construct was made up of all the variables that were loading on factor two (2) all had a common theme that; they represented key features of web 3.0. Hence the eight variables loading under factor (2) were collectively named Web 3.0 features. The eight variables that make up this sub- construct were; predict a service which represents the capability of the platform to predict which service a given user requires or needs based on user demographic data and it has a loading factor of (.888), intelligent services is the next feature which is closely related to the first one but this points more to a platform that will incorporate artificial intelligence in service delivery. It has a loading of (.852), integrated services are the other web 3.0 feature identified. This feature points to a platform that is cohesive and can also incorporate information from various sources before offering a given service. The feature has a loading of (.761). Another feature was the select delivery platform this implies a platform that is ubiquitous in nature and can be 
accessed from any device or platform. The feature has a loading of (.726). Moreover, the research identified the ability of the platform to recognise users as an additional web 3.0 feature. This feature had a loading of (.588) which implies the platform can identify users who are interacting with it. The study also focused on automated services as a key web 3.0 features. This is the ability of a platform to respond to or offer service to a given user without human intervention. The feature had a loading of (.555). Finally, personalisation was identified as a critical web 3.0 feature. It had a loading of (.527). This describes a platform that can offer personalised links and recommendations in response to the request of a user.

The third sub-construct identified as part of Personalized Integrated Service Delivery (PISD) framework was social media management. The sub-construct was made up of all the variables that were loading on factor three (3). All had a common theme around the management of social media platforms. Hence all the five variables loading under factor (3) were collectively named social media management. The five variables that make up this sub- construct were; respond to or act upon issues raised by citizens/ users with loading of (.915). This variable emphasises the significance of responding to or acting upon a request generated by a user. The second variable identified was capturing and storing citizens social media profile data. The variable had a loading (.895). The variable is important since it will facilitate recognition or identification of a citizen while engaging with them and also facilitating personalised service delivery. Thirdly, the research identified under this subcontract was reject/remove inappropriate content as the next variable. This subconstruct had a loading (.845). The variable is necessary so that inappropriate content could be detected/ reported and removed to avoid misuse of the platform. The other variable in this sub-construct was collect and store social media data. The variable had a loading of (.806). This was necessary so that the organisation offering services can retain a database for future reference. Lastly, the study identified a dedicated social media team as a variable under this sub construct. It had a loading of (.504). The variable facilitates smooth delivery of services since there is a team in place to respond to the requests of citizens.

The fourth sub-construct identified as part of Personalized Integrated Service Delivery (PISD) framework was Security. This sub construct had one variable that was loading on factor (5). It had one variable named security. The study notes that the variable represents a key component of any service delivery environment. Therefore, it was retained as part of the personalised integrated service delivery framework and the factor was named security. As stated above the factor had only one variable with a factor loading of (.525).

The fifth sub- construct identified as part of Personalized Integrated Service Delivery (PISD) framework was one-stop-shop for government services. The sub-construct was made up of all the variables that were loading on factor six (6). All the variables had a common theme which featured all government data and services on a common platform. As a result, the three variables loading under factor (6) were collectively named one-stop-shop for government services. The first variable was integration of government data it had a loading of (.621). This implies a platform in which all government data is centrally located and managed as this would ease provision of services. The second variable was device/platform independent services. It had a loading of (.666). The variable describes a platform on which users are able to access the service they require from any device. Lastly, one-stop-shop for services was identified. It has a loading of (.588). Users/citizens can access all the services they require or need on this platform.

The sixth sub-construct identified as part of Personalized Integrated Service Delivery (PISD) framework was External factors. The sub-construct was made up of all the variables that were loading on factor seven (7). The study noted that these variables are not part of the actual platform used to deliver services to users but had an impact or had the potential to affect the way services were being delivered. Therefore, the variables were collectively named external factors. The three variables that made up this subconstruct were: instructional culture with a loading of (.694). This implies that the need to change the culture within institutions when it comes to the use of social media and web 3.0 tools for service delivery to citizens. The second variable under this sub- construct was social media policy. It had a loading of (.570) which emphasises the need for a clear social media policy governing the use of social media for service delivery. The last variable in this sub-construct was training. This variable had a loading of (.528). It emphasises the need for government officials to be trained on how to use social media and web 3.0 technologies for service delivery.

The six factors described above (Services, Web 3.0 features, Social media Management, Security, One Stop Shop and External Factors) form the Personalized Integrated Service Delivery framework (PISD) framework. After this the contribution of each sub-construct to the PISD framework was calculated based on the loading of each variable as derived from the factor analysis. The contributions of these sub-constructs to the PISD framework were calculated using the sum score by factor method [10]. The first step was to consider the individual loadings of all variables that load onto each factor and then compute their average. The result is the Construct Average Loading (CAL) as illustrated in figure 2 below.

Next, the Construct Average Loading (CAL) for the six sub-constructs were summed up $(0.7583+0.6996+0.793$ $+0.525+0.625+0.5973)$. The result was a Total Average Construct Loading (TACL) of 3.9982. This TACL (3.9982) was then used to calculate the effective contribution of each sub-construct towards the PISD framework. When the 
effective weights of the sub-construct are summed up they should add up to one (1). To achieve this, the following computations (CAL / TACL) were carried out;

- $\quad$ Services sub construct $=(0.7583 / 3.9982)=0.1900$

- $\quad$ Web 3.0 features $=(0.6996 / 3.9982)=0.1750$

- $\quad$ Social media management $=(0.793 / 3.9982)=0.1983$

- $\quad$ Secure services $=(0.525 / 3.9982)=0.1313$

- $\quad$ One stop shop for government services $=(0.625 /$ $3.9982)=0.1563$

- $\quad$ External services $(0.5973 / 3.9982)=0.1494$
After calculating the individual contributions for the six sub-constructs which make up the PISD framework and ensuring that they added up to (1.00). The computed weights then fed into the framework. These computed weights were services $(0.1900)$, web 3.0 features $(0.1750)$ social media management $(0.1983)$, secure service (0.1313), one-stop-shop for government services (0.1563) and external factors (0.1494). Figure 1 below illustrates the Personalized Integrated Service Deliver (PISD) framework.

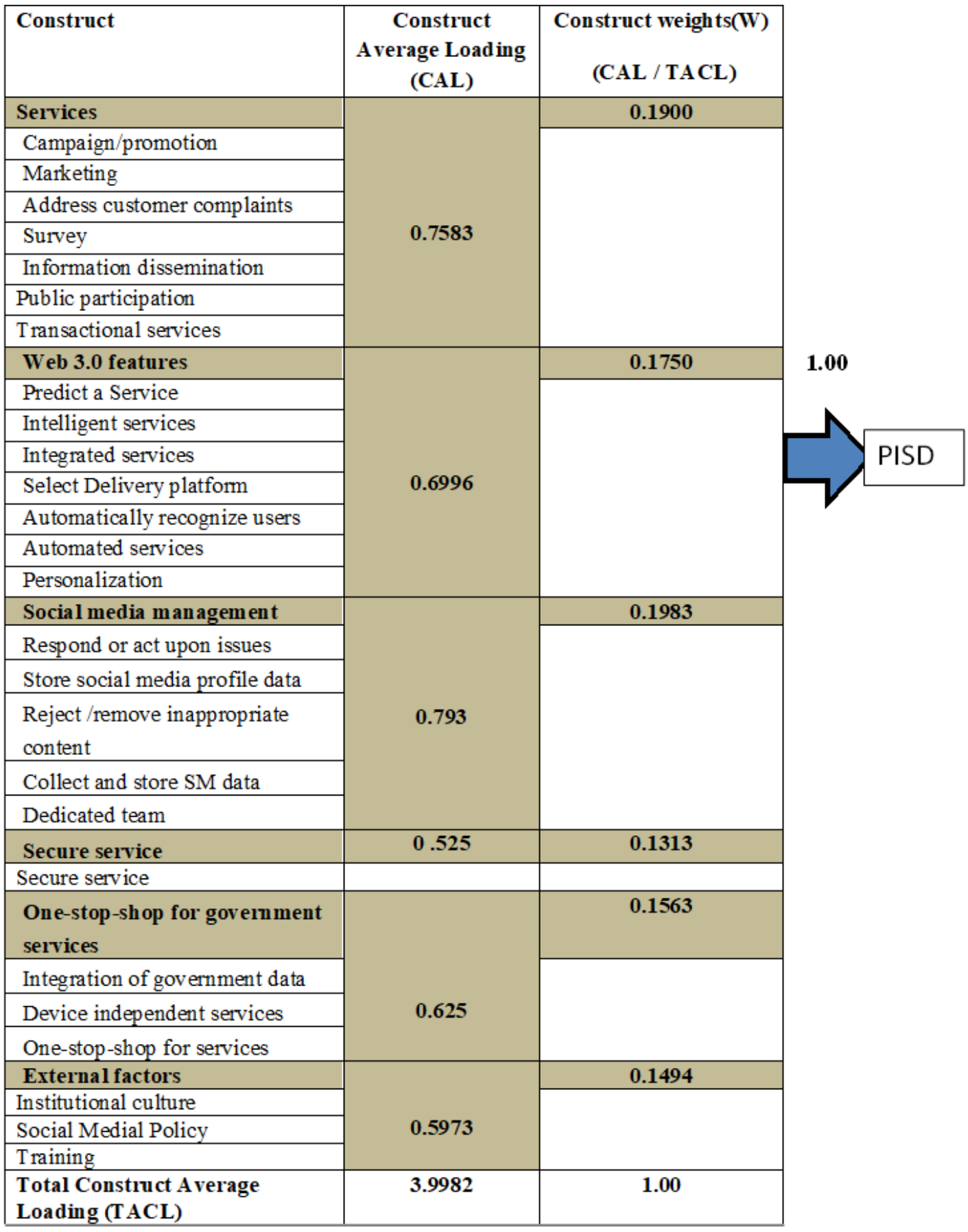

Figure 1. Personalized integrated service delivery framework (PISD) framework 


\section{System Architecture for a Personalized Integrated Service Delivery Environment}

From the discussion above the study developed a PISD framework for Personalised Integrated Services Delivery within government portals. The framework is made up of six factors that is: services, web 3.0 features, social media management, security, one-stop-shop and external factors. Based on these factors, the next step of the study was to develop system architecture (functional structure description.) for a personalised integrated service delivery environment. To develop this system architecture, the study recommends the integration of social media and web 3.0 technologies.

The study argues that the integration of social media and web 3.0 technologies in service delivery within government portals will make government services more personalised, integrated and ubiquitous. Citizens could engage with the government from anywhere and anytime through mobile phones and social media. The government will be able to offer services to citizens in a more accessible, immediate and pro-active way through the integration of social media and web 3.0 technologies.

The study selected social media because of the large number of active users on these platforms as noted by [6] that globally, the total number of social media users is estimated to grow to 3.29 billion by 2022. This will be $42.3 \%$ of the world's population. The Use of web 3.0 in government has been termed as Government 3.0. Web 3.0 was selected to be part of the PISD system architecture since it provides a new paradigm for government operations. It also changes the way the government offers services and interacts with its citizens. Web 3.0 in government aims to provide, customised services for individual citizens. Government 3.0 is a semantic Web-based government that personalises and integrates all government services according to the conditions and preferences of each individual [7].

In the proposed PISD system architecture, the study recommends extensive use of web 3.0 features. This will be achieved through incorporation of an AI-based chatbot developed and embedded within a social media messaging app to automate communication and management of service delivery to citizens. This will allow government to provide eServices that are personalised, integrated, pro-active and ubiquitous. Chatbots today can perform complex tasks like completing proactive transactions due to recent advancements in artificial intelligence and the large quantity of data being available. The private sector is aware of this fact and is taking advantages of AI chatbots to serve customers. Examples of chatbots applications in the private sector include the implementation of virtual assistants (e.g. Siri, Alexa, Google now and Cortana) in various sectors, such as banking (implementing transactions), media (news provision), tourism (booking hotels or tickets), retail, stock market, insurance, gaming agencies, telecommunications, etc. [8].

Based on this, the study argues that chatbots can also be implemented by governments to assist in service delivery to citizens. This is supported by a report from Harvard University that identified six types of government problems, which Artificial Intelligence applications (like AI chatbots) can handle: resource allocation, large datasets, expert shortage, predictable scenarios, procedural and repetitive tasks, diverse data aggregation and summarisation [9]. The government stands to gain a lot by employing AI based chatbots in server's delivery. Some of the accrued benefits will include but not limited to: cost savings, reduction of congestion of citizens at public offices, increased productivity and creation of new jobs.

Figure 2 illustrates the conceptual framework for the PISD system architecture. The proposed system architecture involves the integration of Social Media and Web 3.0 features into government portals for Personalized Integrated Service Delivery. The study recommends the use of an AI based chatbot which will interact with the users (citizens seeking services from government portals). The chatbot will assist citizens to obtain the services they need from government promptly. The chatbot will have the ability to simulate intelligent conversations with citizens using artificial intelligence. Citizens will interact with the chatbot through a conversational interface in written text.

\subsection{High Level Overview of the System Architecture for a Personalized Integrated Service Delivery Environment}

The PISD system architecture will be developed in two phases namely the classifier development, training and testing phase and the chatbot development phase. The classifier development, training and testing phase involves obtaining data from a given portal and using this data to train a classifier that will form a knowledge base. The second phase will involve the development of an AI based chatbot. The knowledge base developed in phase one will consist of information that will support service delivery. The chatbot developed in phase two will use this knowledge base to respond to citizens' requests and provide the services they require. 


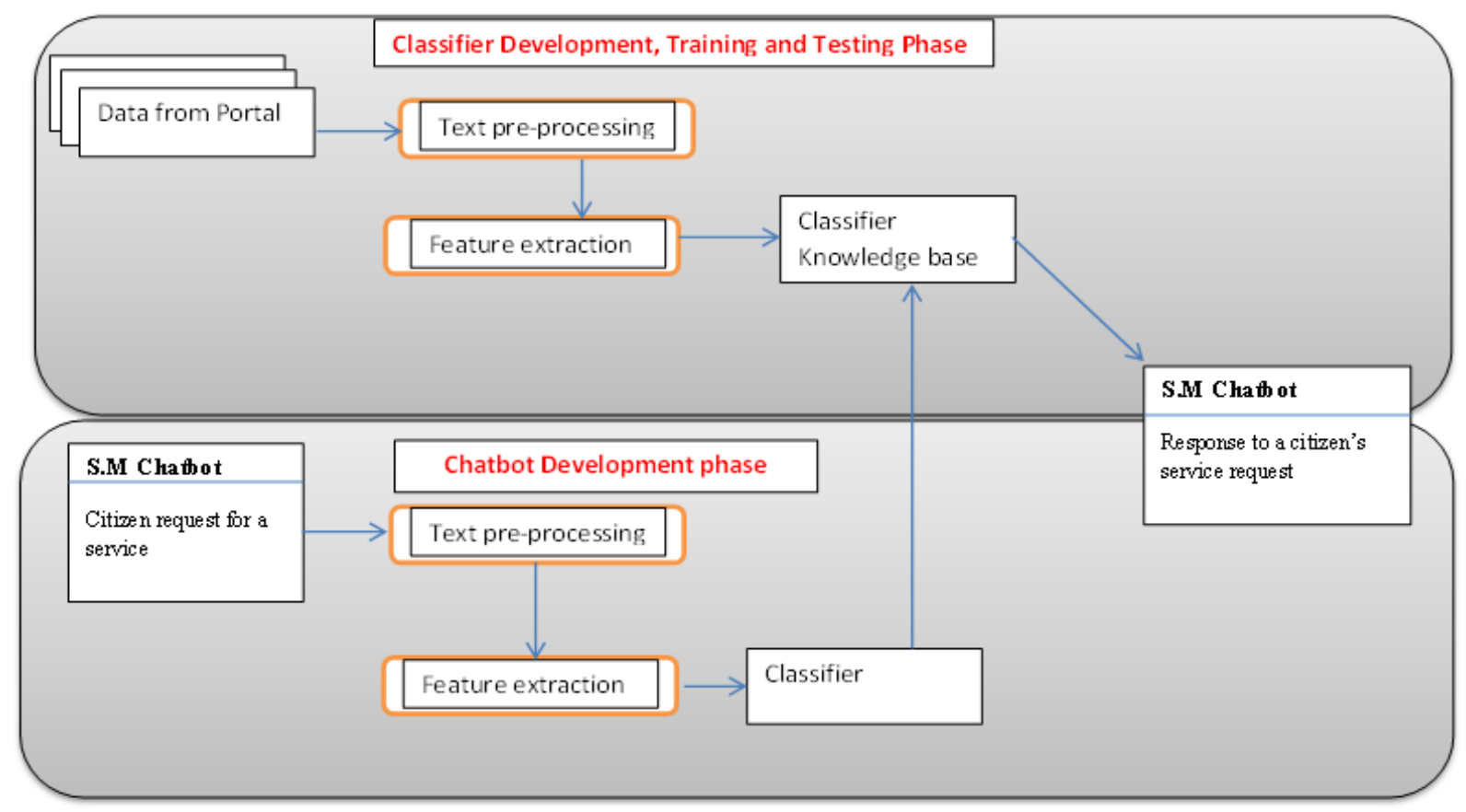

Figure 2. System architecture for an integrated personalised service delivery environment

\subsection{Classifier Development, Training and Testing Phase}

In this phase of the system architecture, the first step is to develop a web crawler that is able to get the data that will form a knowledge base to be used by the chatbot. The web crawler will be run on websites that make up a given government portal. The web clawer will get data on the various services offered through the portal. This data will then be used to train a classifier. However, the data fetched by the crawler will be in an unstructured form and would not be understood by the chatbot. Therefore, the second step in developing the classifier under the training and testing phase is text pre-processing and feature extraction. This is necessary because the data fetched from a given portal would be raw/ unstructured and has to be cleaned to remove noise. Text pre-processing will involve automatic summarisation which is achieved by reducing the raw data collected from a portal to a summary that retains the most significant points of the original data. The main goal of summarisation is to find a representative sub set of the data, which forms a knowledge based for the chatbot. Feature extraction will be achieved through Morphological Analysis which is one of the categories of feature extraction. Morphological analysis involves analysing the individual words represented in the raw data collected from a portal. This mainly consists of tokenisation, remove-stop-word, and stemming- word. First the data is tokenised which means the raw data is treated as a sequence of word strings and splits word by removing punctuations. After this stop-word will be removed. Stop words are words that do not add value or meaning to the data words like "the”, “a”, and “or” were all removed. Removing stop-word is meant to improve the effectiveness and efficiency of text processing because it reduces the number of words in the raw data collected from the portal. The next step of feature extraction is stemming-word which is a linguistic normalisation technique generally used to reduce a word to the root form. The applying” for example can be reduced to its root form which is "apply". Words like "registration, registered and registering" can be reduced to the root form of "register".

Artificial intelligence in the form of supervised machine learning and Natural language processing (NLP) will be employed when creating and training the classifier. The classifier is used to categorise the data that was fetched from the portal. This classified data forms a knowledge base for the chatbot. That is from this classified data (knowledge base) the chatbot will easily identify the service that a citizen is seeking thereby giving a prompt and appropriate response.

\subsection{Chatbot Development Phase}

After getting data from the portal and pre-processing it, extracting features and creating a knowledge base for the chatbot. A chatbot that will make use of this knowledge base to provide services to citizens is then created. Figure 3 below illustrates the steps involved in creating the chatbot. 


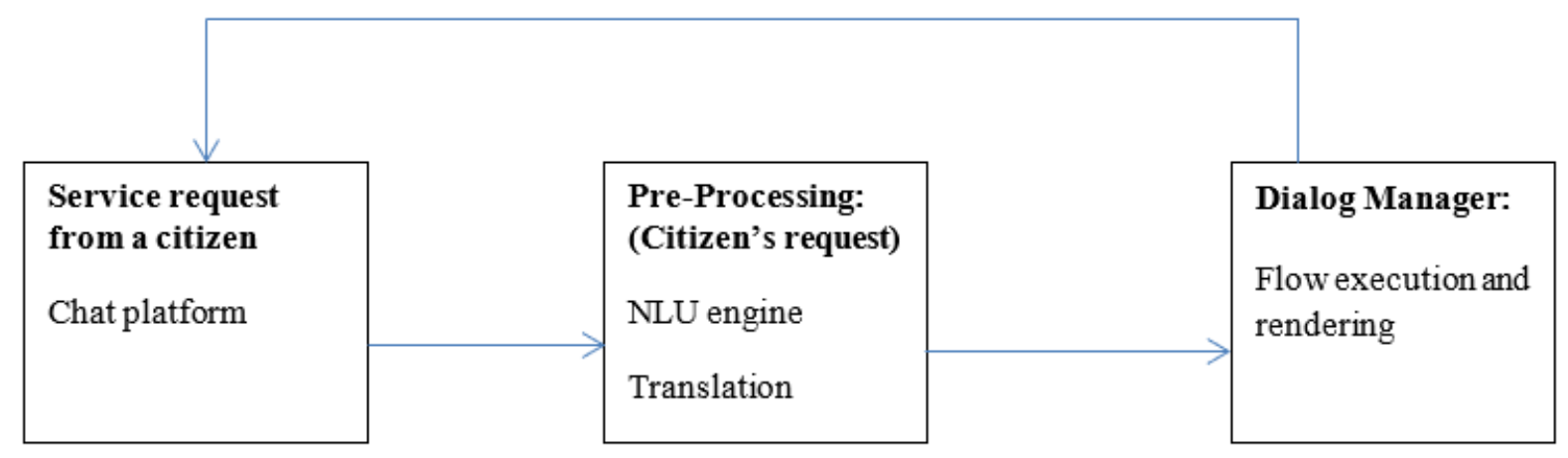

Figure 3. Overview of the Chabot development process

This system architecture is based on the PISD framework and recommends the use of social media as the main channel through which it receives and responds to citizen's requests. The study further recommends the integration of web 3.0 features with social media for effective service delivery. Web 3.0 features will be implemented through an AI chatbot embedded within a social media messaging app. The chatbot will able to handle a citizen's request, provide relevant information to citizens about government services and be the friend citizens would look for (available 24/7) whenever they are seeking information about the government and the services it offers. Hence, citizens will interact with the government through a chatbot that will be hosted within a social medial messaging app.

The chatbot will also ensure security and privacy which is a major concern when it comes to electronic service delivery by government. This was identified as one of the components that make up a Personalised Integrated Service Delivery environment within the PISD framework discussed above. Chatbots have the ability to personalise every conversation to the core without becoming intrusive or invading privacy also opposed to social media group messaging apps. Therefore, this study recommends the use of a chatbot developed, managed and fully owned by government agencies instead rather than the use of third party messaging platforms from Facebook or twitter. The main reason for recommending use of chatbots is because chatbots will ensure security and the privacy of the information being passed through this system framework. The chatbot will also ensure that Personal Identification Information (PII), data and general information that is being exchanged between government and citizens will be managed, controlled and owned by the government.

The chatbot will incorporate an efficient queuing mechanism that is able to process incoming requests from citizens and handle outgoing responses sequentially. The chatbot development platform selected will be installed and configured individually and locally, which means that the government would have full control over the data that is transmitted between the bot and the chat platforms. The chatbot will make use of Artificial Intelligence in order to understand citizen's requests and respond to them accordingly. Once the chatbot receives a request from a citizen, it makes use of Natural Language Understanding (NLU) to pre-process the request into a form that the bot can understand. NLU engine involves the bot processing the messages received from the chat platform, which is usually in unstructured text, and transforming them into structured data that the chatbot will be able to work with.

The main tasks of this NLU engine which is a component of the AI based chatbot are: intent recognition: recognising what the citizen is requesting for, entity extraction: extracting structured information from the citizens' request, for example, type of service requested, names, institution and more, slot tagging: identify necessary parameters to accomplish a given task, language identification: knowing in which language the user is writing (limited to English only). The above tasks will be done by the NLU engine and are very critical to the creation of more natural and satisfying conversations with the chatbot.

Once the chatbot has received a citizen's request from the chat platform it transforms the request into structured data using the NLU engine and understands the citizen's request. The next step will involve the Dialog Manager (or $\mathrm{DM})$ component. The main goal for the DM component is to use the knowledge base that was created during the training and testing phase to determine how the bot should act in order to respond to a citizen's request. For example, what the bot should do or say next, (delivery of a service). The study recommends the use of a statistical based decisions making Dialog Manager and an extensible Visual Flow Editor instead of a group of "If" and "Else" statements, owing to the unpredictability of natural language and the fact that "If" and "Else" statements, are not effective as they do not scale well in practice. Therefore, the proposed PISD system architecture will be able to receive a citizens' request through the chatbot embedded within a social media messaging app (belonging to the official government social media account). After which the chatbot will make use of Natural Language Understanding (NLU) engine to comprehend the citizen's request and finally use the Dialog flow component to access the knowledge base already developed and respond appropriately to the citizen's request. 


\section{Conclusions}

The study concludes that web portals play an important role in service delivery to citizens especially in developing countries like Kenya web 3.0 and Social Media technologies have the potential to transform the way the government interacts and delivers services to its citizens. If only the government took advantage of the numerous features of web 3.0 tools and social media technologies. This paper has considered how web 3.0 and social media technologies can be incorporated within government portals for Personalized Integrated Service Delivery. The study was guided by opinions from a team of experts who are involved in developing, integrating, and maintaining portal and social media services for the Kenya government eCitizen portal.

A Personalised Integrated Service Delivery (PISD) framework was developed. The study also described a Personalized Integrated Service Delivery System architecture based on the PISD framework. The proposed system architecture employs an AI chatbot embedded within a social media messaging app. The PISD framework and the proposed system architecture are expected to guide governments on how to fully utilise web 3.0 and social media technologies in services delivery.

\section{REFERENCES}

[1] United Nations. (2018). United Nations E-Government Survey 2018. New York: United Nations.
[2] United Nations, U. (2016). United Nations E-Government Survey 2016: E-Government in Support of Sustainable Development. United Nations.

[3] ecitizen. (2014). Retrieved 11 5, 2019, from ecitizen: https://ecitizen.go.ke

[4] Kaiser, H. (1960). The application of electronic computers to factor analysis. Educational and Psychological Measurement, 141-151.

[5] Pett, M., \& Lackey, R. (2003). Making Sense of Factor Analysis: The use of factor analysis for instrument development in health care research. California: Sage Publications Inc.

[6] eMarketer. (2018). Social Network Users and Penetration in Worldwide. Retrieved from eMarketer:https://tinyurl.com/y cr2d3v9.

[7] Cho, J.-S. (2017). Evolution of e-government: Transparency, competency, and service oriented government with Korean government 3.0. Journal of Business and Retail Management Research (JBRMR), Vol. 12 Issue 1.

[8] Aggeliki, A., E, L., Nikos, K., \& Charalabidis, Y. (2018). Transforming the communication between citizens and government through AI-guided chatbots. Government Information Quarterly.

[9] Mehr, H. (2017). Artificial Intelligence for Citizen Services and Government Artificial Intelligence for Citizen Services and Government artificial intelligence for citizen services and government. Retrieved from https://ash.harvard.edu/file s/ash/files/artificial_intelligence_for_citizen_services.pdf.

[10] Hair, J., W.C., B., B.J., B., R.E., A., \& R.L., T. (2006). Multivariate data analysis. Upper Saddle River: Pearson Prentic 